\title{
q-Analog of Gelfand-Graev Basis for the Noncompact Quantum Algebra $U_{q}(u(n, 1))^{\star}$
}

Raisa M. ASHEROVA ${ }^{\dagger}$, Čestmír BURDÍK ${ }^{\ddagger}$, Miloslav HAVLÍČEK ${ }^{\ddagger}$, Yuri F. SMIRNOV ${ }^{\dagger \S}$ and Valeriy N. TOLSTOY ${ }^{\dagger \ddagger}$

$\dagger$ Institute of Nuclear Physics, Moscow State University, 119992 Moscow, Russia

E-mail: raya.acherova@gmail.com, tolstoy@nucl-th.sinp.msu.ru

$¥$ Department of Mathematics, Faculty of Nuclear Sciences and Physical Engineering, Czech Technical University in Prague, Trojanova 13, 12000 Prague 2, Czech Republic

E-mail: burdik@kmlinux.fjfi.cvut.cz, miloslav.havlicek@fjfi.cvut.cz

\section{$\S$ Deceased}

Received November 05, 2009, in final form January 15, 2010; Published online January 26, 2010 doi:10.3842/SIGMA.2010.010

\begin{abstract}
For the quantum algebra $U_{q}(\mathfrak{g l}(n+1))$ in its reduction on the subalgebra $U_{q}(\mathfrak{g l}(n))$ an explicit description of a Mickelsson-Zhelobenko reduction $Z$-algebra $Z_{q}(\mathfrak{g l}(n+1), \mathfrak{g l}(n))$ is given in terms of the generators and their defining relations. Using this $Z$-algebra we describe Hermitian irreducible representations of a discrete series for the noncompact quantum algebra $U_{q}(u(n, 1))$ which is a real form of $U_{q}(\mathfrak{g l}(n+1))$, namely, an orthonormal Gelfand-Graev basis is constructed in an explicit form.
\end{abstract}

Key words: quantum algebra; extremal projector; reduction algebra; Shapovalov form; noncompact quantum algebra; discrete series of representations; Gelfand-Graev basis

2010 Mathematics Subject Classification: 17B37; 81R50

\section{Introduction}

In 1950, I.M. Gelfand and M.L. Tsetlin [1] proposed a formal description of finite-dimensional irreducible representations (IR) for the compact Lie algebra $u(n)$. This description is a generalization of the results for $u(2)$ and $u(3)$ to the $u(n)$ case. It is the following. In the IR space of $u(n)$ there is a orthonormal basis which is numerated by the following formal schemes:

$$
\left(\begin{array}{ccccccccc}
m_{1 n} & & m_{2 n} & & \ldots & & m_{n-1, n} & & m_{n n} \\
& m_{1, n-1} & & m_{2, n-1} & & \ldots & & m_{n-1, n-1} & \\
& & \ldots & & \ldots & & \ldots & &
\end{array}\right),
$$

where all numbers $m_{i j}(1 \leq i \leq j \leq n)$ are nonnegative integers and they satisfy the standard inequalities, "between conditions":

$$
m_{i j+1} \geq m_{i j} \geq m_{i+1 j+1} \quad \text { for } 1 \leq i \leq j \leq n-1 .
$$

The first line of this scheme is defined by the components of the highest weight of $u(n)$ IR, the second line is defined by the components of the highest weight of $u(n-1)$ IR and so on.

${ }^{\star}$ This paper is a contribution to the Proceedings of the XVIIIth International Colloquium on Integrable Systems and Quantum Symmetries (June 18-20, 2009, Prague, Czech Republic). The full collection is available at http://www.emis.de/journals/SIGMA/ISQS2009.html 
Later this basis was constructed in many papers (see, e.g., $[2,3,4]$ ) by using one-step lowering and raising operators.

In 1965, I.M. Gelfand and M.I. Graev [5], using analytic continuation of the results for $u(n)$, obtained some results for noncompact Lie algebra $u(n, m)$. It was shown that some class of Hermitian IR of $u(n, m)$ is characterized by an "extremal weight" parametrized by a set of integers $m_{N}=\left(m_{1 N}, m_{2 N}, \ldots, m_{N N}\right)(N=n+m)$ such that $m_{1 N} \geq m_{2 N} \geq \cdots \geq m_{N N}$, and by a representation type which is defined by a partition of $n$ in the sum of two nonnegative integers $\alpha$ and $\beta, n=\alpha+\beta$ (also see [6]).

For simplicity we consider the case $u(2,1)$. In this case we have three types of schemes

$$
\begin{aligned}
& \left(\begin{array}{ccccc} 
& m_{13} & & m_{23} & m_{33} \\
m_{12} & & m_{22} & &
\end{array}\right) \quad \text { for }(\alpha, \beta)=(2,0) \text {, }
\end{aligned}
$$

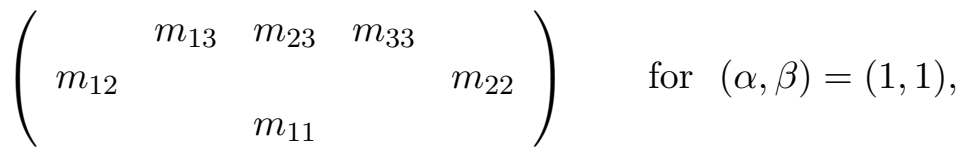

$$
\begin{aligned}
& \left(\begin{array}{ccccc}
m_{13} & m_{23} & & m_{33} & \\
& & m_{12} & & m_{22} \\
& & & m_{11} &
\end{array}\right) \quad \text { for }(\alpha, \beta)=(0,2) \text {. }
\end{aligned}
$$

The numbers $m_{i j}$ of the first scheme satisfy the following inequalities

$$
m_{12} \geq m_{13}+1, \quad m_{13}+1 \geq m_{22} \geq m_{23}+1, \quad m_{12} \geq m_{11} \geq m_{22} .
$$

The numbers $m_{i j}$ of the second scheme satisfy the following inequalities

$$
m_{12} \geq m_{13}+1, \quad m_{33}-1 \geq m_{22}, \quad m_{12} \geq m_{11} \geq m_{22} .
$$

The numbers of the third scheme satisfy the following inequalities

$$
m_{23}-1 \geq m_{12} \geq m_{33}-1, \quad m_{33}-1 \geq m_{22}, \quad m_{12} \geq m_{11} \geq m_{22} .
$$

Construction of the Gelfand-Graev basis for $u(n, m)$ in terms of one-step lowering and raising operators is more complicated than in the compact case $u(n+m)$.

In 1975, T.J. Enright and V.S. Varadarajan [7] obtained a classification of discrete series of noncompact Lie algebras. Later it was proved by A.I. Molev [8] that in the case of $u(n, m)$ the Gelfand-Graev modules are part of the Enright-Varadarajan modules and Molev constructed the Gelfand-Graev basis for $u(n, m)$ in terms of the Mickelsson S-algebra [9].

A goal of this work is to obtain analogous results for the noncompact quantum algebra $U_{q}(u(n, m))$. Since the general case is very complicated we at first consider the case $U_{q}(u(n, 1))$. The special case $U_{q}(u(2,1))$ was considered in [10,11]. It should be noted that the principal series representations of $U_{q}(u(n, 1))$ were studied in [12] and a classification of unitary highest weight modules of $U_{q}(u(n, 1))$ was considered in [13].

\section{Quantum algebra $U_{q}(\mathfrak{g l}(N))$ and its noncompact real forms $U_{q}(u(n, m))(n+m=N)$}

The quantum algebra $U_{q}(\mathfrak{g l}(N))$ is generated by the Chevalley elements $q^{ \pm e_{i i}}(i=1, \ldots, N)$, $e_{i, i+1}, e_{i+1, i}(i=1,2, \ldots, N-1)$ with the defining relations $[14,15]$ :

$$
q^{e_{i i}} q^{-e_{i i}}=q^{-e_{i i}} q^{e_{i i}}=1,
$$




$$
\begin{aligned}
& q^{e_{i i}} q^{e_{j j}}=q^{e_{j j}} q^{e_{i i}}, \\
& q^{e_{i i}} e_{j k} q^{-e_{i i}}=q^{\delta_{i j}-\delta_{i k}} e_{j k} \quad(|j-k|=1), \\
& {\left[e_{i, i+1}, e_{j+1, j}\right]=\delta_{i j} \frac{q^{e_{i i}-e_{i+1, i+1}}-q^{e_{i+1, i+1}-e_{i i}}}{q-q^{-1}},} \\
& {\left[e_{i, i+1}, e_{j, j+1}\right]=0 \quad \text { for }|i-j| \geq 2,} \\
& {\left[e_{i+1, i}, e_{j+1, j}\right]=0 \quad \text { for }|i-j| \geq 2,} \\
& {\left[\left[e_{i, i+1}, e_{j, j+1}\right]_{q}, e_{j, j+1}\right]_{q}=0 \quad \text { for }|i-j|=1,} \\
& {\left[\left[e_{i+1, i}, e_{j+1, j}\right]_{q}, e_{j+1, j}\right]_{q}=0 \quad \text { for }|i-j|=1,}
\end{aligned}
$$

where $\left[e_{\beta}, e_{\gamma}\right]_{q}$ denotes the $q$-commutator:

$$
\left[e_{\beta}, e_{\gamma}\right]_{q}:=e_{\beta} e_{\gamma}-q^{(\beta, \gamma)} e_{\gamma} e_{\beta} .
$$

The definition of a quantum algebra also includes operations of a comultiplication $\Delta_{q}$, an antipode $S_{q}$, and a co-unit $\epsilon_{q}$. Explicit formulas of these operations will not be used in our later calculations and they are not given here.

Let $\varepsilon_{i}(i=1,2, \ldots, N)$ be a dual basis to the Cartan basis $e_{i i}(i=1,2, \ldots, N), \varepsilon_{i}\left(e_{j j}\right)=$ $\left(\varepsilon_{i}, \varepsilon_{j}\right)=\delta_{i j}$. In terms of the orthonormal basis vectors $\varepsilon_{i}$ the positive root system $\Delta_{+}$of $\mathfrak{g l}(N)$ $\left(U_{q}(\mathfrak{g l}(N))\right)$ is presented as follows:

$$
\Delta_{+}=\left\{\varepsilon_{i}-\varepsilon_{j} \mid 1 \leq i<j \leq N\right\},
$$

where $\varepsilon_{i}-\varepsilon_{i+1}(i=1,2, \ldots, N-1)$ are the simple roots.

Since for construction of the composite root vectors $e_{i j}:=e_{\varepsilon_{i}-\varepsilon_{j}}(|i-j| \geq 2)$ of the quantum algebra $U_{q}(\mathfrak{g l}(N))$ we need to use the notation of the normal ordering in the positive root system $\Delta_{+}$, we recall this notation. We say that the system $\Delta_{+}$is written in normal (convex) ordering, $\vec{\Delta}_{+}$, if each positive composite root $\varepsilon_{i}-\varepsilon_{j}=\left(\varepsilon_{i}-\varepsilon_{k}\right)+\left(\varepsilon_{k}-\varepsilon_{j}\right)(i \leq k \leq j)$ is located between its components $\varepsilon_{i}-\varepsilon_{k}$ and $\varepsilon_{k}-\varepsilon_{j}$. It means that in the normal ordering system $\vec{\Delta}_{+}$we have either

$$
\ldots, \varepsilon_{i}-\varepsilon_{k}, \ldots, \varepsilon_{i}-\varepsilon_{j}, \ldots, \varepsilon_{k}-\varepsilon_{j}, \ldots
$$

or

$$
\ldots, \varepsilon_{k}-\varepsilon_{j}, \ldots, \varepsilon_{i}-\varepsilon_{j}, \ldots, \varepsilon_{i}-\varepsilon_{k}, \ldots
$$

There are many normal orderings in the root system $\Delta_{+}=\Delta_{+}(\mathfrak{g l}(N))$, more than $(N-1)$ ! for $N>3$. To be definite, we fix the following normal ordering (see $[14,15]$ )

$$
\begin{aligned}
& \varepsilon_{1}-\varepsilon_{2} \prec \varepsilon_{1}-\varepsilon_{3} \prec \varepsilon_{2}-\varepsilon_{3} \prec \varepsilon_{1}-\varepsilon_{4} \prec \varepsilon_{2}-\varepsilon_{4} \prec \varepsilon_{3}-\varepsilon_{4} \prec \cdots \prec \\
& \varepsilon_{1}-\varepsilon_{k} \prec \varepsilon_{2}-\varepsilon_{k} \prec \cdots \prec \varepsilon_{k-1}-\varepsilon_{k} \prec \cdots \prec \varepsilon_{1}-\varepsilon_{N} \prec \varepsilon_{2}-\varepsilon_{N} \prec \cdots \prec \varepsilon_{N-1}-\varepsilon_{N} .
\end{aligned}
$$

According to this ordering, we determine the composite root vectors $e_{i j}$ for $|i-j| \geq 2$ as follows:

$$
e_{i j}:=\left[e_{i k}, e_{k j}\right]_{q^{-1}}, \quad e_{j i}:=\left[e_{j k}, e_{k i}\right]_{q},
$$

where $1 \leq i<k<j \leq N$. It should be stressed that the structure of the composite root vectors does not depend on the choice of the index $k$ on the right-hand side of the definition (2.10). In particular, we have

$$
\begin{aligned}
e_{i j} & :=\left[e_{i, i+1}, e_{i+1, j}\right]_{q^{-1}}=\left[e_{i, j-1}, e_{j-1, j}\right]_{q^{-1}}, \\
e_{j i} & :=\left[e_{j, i+1}, e_{i+1, i}\right]_{q}=\left[e_{j, j-1}, e_{j-1, i}\right]_{q},
\end{aligned}
$$

where $2 \leq i+1<j \leq N$. 
Using these explicit constructions and defining relations (2.1)-(2.8) for the Chevalley basis it is not hard to calculate the following relations between the Cartan-Weyl generators $e_{i j}(i, j=$ $1,2, \ldots, N)$ :

$$
\begin{aligned}
& q^{e_{k k}} e_{i j} q^{-e_{k k}}=q^{\delta_{k i}-\delta_{k j}} e_{i j} \quad(1 \leq i, j, k \leq N), \\
& {\left[e_{i j}, e_{j i}\right]=\frac{q^{e_{i i}-e_{j j}}-q^{e_{j j}-e_{i i}}}{q-q^{-1}} \quad(1 \leq i<j \leq N),} \\
& {\left[e_{i j}, e_{k l}\right]_{q^{-1}}=\delta_{j k} e_{i l} \quad(1 \leq i<j \leq k<l \leq N),} \\
& {\left[e_{i k}, e_{j l}\right]_{q^{-1}}=\left(q-q^{-1}\right) e_{j k} e_{i l} \quad(1 \leq i<j<k<l \leq N),} \\
& {\left[e_{j k}, e_{i l}\right]_{q^{-1}}=0 \quad(1 \leq i \leq j<k<l \leq N),} \\
& {\left[e_{i k}, e_{j k}\right]_{q^{-1}}=0 \quad(1 \leq i<j<k \leq N),} \\
& {\left[e_{k l}, e_{j i}\right]=0 \quad(1 \leq i<j \leq k<l \leq N),} \\
& {\left[e_{i l}, e_{k j}\right]=0 \quad(1 \leq i<j<k<l \leq N),} \\
& {\left[e_{j i}, e_{i l}\right]=e_{j l} q^{e_{i i}-e_{j j}} \quad(1 \leq i<j<l \leq N),} \\
& {\left[e_{k l}, e_{l i}\right]=e_{k i} q^{e_{k k}-e_{l l}} \quad(1 \leq i<k<l \leq N),} \\
& {\left[e_{j l}, e_{k i}\right]=\left(q^{-1}-q\right) e_{k l} e_{j i} q^{e_{j j}-e_{k k}} \quad(1 \leq i<j<k<l \leq N) .}
\end{aligned}
$$

If we apply the Cartan involution $\left(e_{i j}^{\star}=e_{j i}, q^{\star}=q^{-1}\right)$ to the formulas (2.12)-(2.22), we get all relations between the elements of the Cartan-Weyl basis.

The explicit formula for the extremal projector for $U_{q}(\mathfrak{g l}(N))$, corresponding to the fixed normal ordering (2.9), has the form $[14,15]$

$$
\begin{aligned}
p\left(U_{q}(\mathfrak{g l} l(N))\right. & =p\left(U_{q}(\mathfrak{g l}(N-1))\left(p_{1 N} p_{2 N} \cdots p_{N-2, N} p_{N-1, N}\right)\right. \\
& =p_{12}\left(p_{13} p_{23}\right) \cdots\left(p_{1 k} \cdots p_{k-1, k}\right) \cdots\left(p_{1 N} \cdots p_{N-1, N}\right),
\end{aligned}
$$

where the elements $p_{i j}(1 \leq i<j \leq N)$ are given by

$$
p_{i j}=\sum_{r=0}^{\infty} \frac{(-1)^{r}}{[r] !} \varphi_{i j, r} e_{i j}^{r} e_{j i}^{r}, \quad \varphi_{i j, r}=q^{-(j-i-1) r}\left\{\prod_{s=1}^{r}\left[e_{i i}-e_{j j}+j-i+s\right]\right\}^{-1} .
$$

Here and elsewhere the symbol $[x]$ is given as follows

$$
[x]=\frac{q^{x}-q^{-x}}{q-q^{-1}} .
$$

The extremal projector $p:=p\left(U_{q}(\mathfrak{g l}(N))\right.$ satisfies the relations:

$$
e_{i, i+1} p=p e_{i+1, i}=0 \quad(1 \leq i \leq N-1), \quad p^{2}=p .
$$

The extremal projector $p$ belongs to the Taylor extension $T U_{q}(\mathfrak{g l}(N))$ of the quantum algebras $U_{q}(\mathfrak{g l}(N))$. The Taylor extension $T U_{q}(\mathfrak{g l}(N))$ is an associative algebra generated by formal Taylor series of the form

$$
\sum_{\{\tilde{r}\},\{r\}} C_{\{\tilde{r}\},\{r\}}\left(q^{e_{11}}, \ldots, q^{e_{N N}}\right) e_{21}^{\tilde{r}_{12}} e_{31}^{\tilde{r}_{13}} e_{32}^{\tilde{r}_{23}} \cdots e_{N, N-1}^{\tilde{r}_{N-1, N}} e_{12}^{r_{12}} e_{13}^{r_{13}} e_{23}^{r_{23}} \cdots e_{N-1, N}^{r_{N-1, N}}
$$

provided that nonnegative integers $\tilde{r}_{12}, \tilde{r}_{13}, \tilde{r}_{23}, \ldots, \tilde{r}_{N-1, N}$ and $r_{12}, r_{13}, r_{23}, \ldots, r_{N-1, N}$ are subject to the constraints

$$
\left|\sum_{i<j} \tilde{r}_{i j}-\sum_{i<j} r_{i j}\right| \leq \mathrm{const}
$$


for each formal series and the coefficients $C_{\{\tilde{r}\},\{r\}}\left(q^{e_{11}}, \ldots, q^{e_{N N}}\right)$ are rational functions of the $q$-Cartan elements $q^{e_{i i}}$. The quantum algebra $U_{q}(\mathfrak{g l} l(N))$ is a subalgebra of the Taylor extension $T U_{q}(\mathfrak{g l}(N)), U_{q}(\mathfrak{g l}(N)) \subset T U_{q}(\mathfrak{g l}(N))$.

We consider, on the quantum algebra $U_{q}(\mathfrak{g l}(N))$, two real forms: compact and noncompact.

The compact quantum algebra $U_{q}(\mathfrak{u}(N))$ can be considered as the quantum algebra $U_{q}(\mathfrak{g l}(N))$ $(N=n+m)$ endowed with the additional Cartan involution $\star$ :

$$
\begin{array}{lc}
h_{i}^{\star}=h_{i} \quad \text { for } \quad i=1,2, \ldots, N, & \\
e_{i, i+1}^{\star}=e_{i+1, i}, & e_{i+1, i}^{\star}=e_{i, i+1} \\
q^{\star}=q \quad \text { or } \quad & q^{\star}=q^{-1} .
\end{array} \quad \text { for } \quad 1 \leq i \leq N-1,
$$

Thus we have two compact real forms: with real $q\left(q^{\star}=q\right)$ and with circular $q\left(q^{\star}=q^{-1}\right)$. In the case of the circular $q$ the Cartan-Weyl basis $e_{i j}(i, j=1,2, \ldots, N)$ constructed by formulas (2.10) is $\star$-invariant, i.e. $e_{i j}^{\star}=e_{j i}$ for all $1 \leq i, j \leq N$. In the case of the real $q$ this Cartan-Weyl basis is not $\star$-invariant, since the basis vectors satisfy the relations $e_{i j}^{\star}=e_{j i}^{\prime}$ for $|i-j| \geq 2$ where the root vectors $e_{j i}^{\prime}$ are obtained from $(2.10)$ by the replacement $q^{ \pm 1} \rightarrow q^{\mp 1}$.

It is reasonable to consider the real compact form on the Taylor extension $T U_{q}(\mathfrak{g l}(N))$. In particular, it should be noted that

$$
p^{\star}=p \quad \text { for real and circular } q .
$$

This property is a direct consequence of a uniqueness theorem for the extremal projector, which states that equations (2.25) have a unique nonzero solution in the space of the Taylor extension $T U_{q}(\mathfrak{g l}(N))$ and this solution does not depend on the choice of normal ordering and on the replacement $q^{ \pm 1} \rightarrow q^{\mp 1}$ in formulas (2.10).

The noncompact quantum algebra $U_{q}(\mathfrak{u}(n, m))$ can be considered as the quantum algebra $U_{q}(\mathfrak{g l}(N))(N=n+m)$ endowed with the additional Cartan involution $*$ :

$$
\begin{aligned}
& h_{i}^{*}=h_{i} \quad \text { for } \quad i=1,2, \ldots, N, \\
& e_{i, i+1}^{*}=e_{i+1, i}, \quad e_{i+1, i}^{*}=e_{i, i+1} \quad \text { for } \quad 1 \leq i \leq N-1, \quad i \neq n, \\
& e_{n, n+1}^{*}=-e_{n+1, n}, \quad e_{n+1, n}^{*}=-e_{n, n+1}, \\
& q^{*}=q \quad \text { or } \quad q^{*}=q^{-1} .
\end{aligned}
$$

We also have two noncompact real forms: with real $q\left(q^{\star}=q\right)$ and with circular $q\left(q^{\star}=q^{-1}\right)$. Below we will consider the real form $U_{q}(\mathfrak{u}(n, 1))$, i.e. the case $N=n+1$.

\section{The reduction algebra $Z_{q}(\mathfrak{g l}(n+1), \mathfrak{g l}(n))$}

In the linear space $T U_{q}(\mathfrak{g l}(n+1))$ we separate out a subspace of "two-sided highest vectors" with respect to the subalgebra $U_{q}(\mathfrak{g l} l(n)) \subset U_{q}(\mathfrak{g l} l(n+1))$, i.e.

$$
\tilde{Z}_{q}(\mathfrak{g l}(n+1), \mathfrak{g l}(n))=\left\{x \in T U_{q}(\mathfrak{g l}(n+1)) \mid e_{i, i+1} x=x e_{i+1, i}=0,1 \leq i \leq n-1\right\} .
$$

It is evident that if $x \in \tilde{Z}_{q}(\mathfrak{g l}(n+1), \mathfrak{g l}(n))$ then

$$
x=p x p,
$$

where $p:=p\left(U_{q}(\mathfrak{g l}(n))\right.$. Again, using the annihilation properties of the projection operator $p$ we have that any vector $x \in \tilde{Z}_{q}(\mathfrak{g l}(n+1), \mathfrak{g l}(n))$ can be presented in the form of a formal Taylor series on the following monomials

$$
p e_{n+1,1}^{r_{1}^{\prime}} \cdots e_{n+1, n}^{r_{n}^{\prime}} e_{n, n+1}^{r_{n}} \cdots e_{1, n+1}^{r_{1}} p .
$$


It is evident that $\tilde{Z}_{q}(\mathfrak{g l}(n+1), \mathfrak{g l}(n))$ is a subalgebra in $T U_{q}(\mathfrak{g l}(n+1))$. We consider a subalgebra $Z_{q}(\mathfrak{g l}(n+1), \mathfrak{g l}(n))$ generated by finite series on monomials $(3.1)$.

We set

$$
z_{0}:=p, \quad z_{i}:=p e_{i, n+1} p, \quad z_{-i}:=p e_{n+1, i} p \quad(i=1,2, \ldots, n) .
$$

Theorem 1. The elements $z_{i}(i=0, \pm 1, \pm 2, \ldots, \pm n)$ generate the unital associative algebra $Z_{q}(\mathfrak{g l}(n+1), \mathfrak{g l}(n))$ and satisfy the following relations

$$
\begin{aligned}
& z_{0} z_{i}=z_{i} z_{0}=z_{i} \quad \text { for } \quad i=0, \pm 1, \pm 2, \ldots, \pm n, \\
& z_{i} z_{-j}=z_{-j} z_{i} \quad \text { for } \quad 1 \leq i, j \leq n, \quad i \neq j, \\
& z_{i} z_{j}=z_{j} z_{i} \frac{\left[\varphi_{i j}+1\right]}{\left[\varphi_{i j}\right]} \quad \text { for } \quad 1 \leq i<j \leq n, \\
& z_{-i} z_{-j}=z_{-j} z_{-i} \frac{\left[\varphi_{i j}\right]}{\left[\varphi_{i j}+1\right]} \quad \text { for } \quad 1 \leq i<j \leq n,
\end{aligned}
$$

and

$$
z_{i} z_{-i}=\sum_{j=1}^{n} B_{i j} z_{-j} z_{j}+\gamma_{i} z_{0} \quad \text { for } \quad i=1,2, \ldots, n
$$

where

$$
\begin{aligned}
& B_{i j}=-\frac{b_{i}^{-} b_{j}^{+}}{\left[\varphi_{i j}-1\right]}, \quad \gamma_{i}=\left[\varphi_{i, n+1}-1\right] b_{i}^{-}, \\
& b_{i}^{ \pm}=\prod_{s=i+1}^{n} \frac{\left[\varphi_{i s} \pm 1\right]}{\left[\varphi_{i s}\right]}, \quad \varphi_{i j}=e_{i i}-e_{j j}+j-i .
\end{aligned}
$$

Remark 1. The relations (3.2) state that the element $z_{0}$ is an algebraic unit in $Z_{q}(\mathfrak{g l}(n+1)$, $\mathfrak{g l}(n))$.

A proof of the theorem can be obtained by direct calculations using the explicit form of extremal projector $(2.23),(2.24)$.

It should be noted that the theorem was proved by V.N.T. as early as 1989 but it has not been published up to now, however, the results of the theorem were used for construction of the Gelfand-Tsetlin basis for the compact quantum algebra $U_{q}(u(n))[14,15]$.

For construction and study of the discrete series of the noncompact quantum algebra $U_{q}(\mathfrak{u}(n, 1)$ we need other relations than (3.6). The system (3.6) expresses the elements $z_{i} z_{-i}$ in terms of the elements $z_{-i} z_{i}(i=1,2, \ldots, n)$ but we would like to express the elements $z_{-1} z_{1}, \ldots, z_{-\alpha} z_{\alpha}$, $z_{\alpha+1} z_{-\alpha-1}, \ldots, z_{n} z_{-n}$ in terms of the elements $z_{1} z_{-1}, \ldots, z_{\alpha} z_{-\alpha}, z_{-\alpha-1} z_{\alpha+1}, \ldots, z_{-n} z_{n}$ for $\alpha=$ $0,1, \ldots, n .{ }^{1,2}$ These relations are given by the proposition.

Proposition 1. The elements $z_{-1} z_{1}, \ldots, z_{-\alpha} z_{\alpha}, z_{\alpha+1} z_{-\alpha-1}, \ldots, z_{n} z_{-n}$ are expressed in terms of the elements $z_{1} z_{-1}, \ldots, z_{\alpha} z_{-\alpha}, z_{-\alpha-1} z_{\alpha+1}, \ldots, z_{-n} z_{n}$ by the formulas

$$
\begin{array}{ll}
z_{-i} z_{i}=\sum_{j=1}^{\alpha} B_{i j}^{(\alpha)} z_{j} z_{-j}+\sum_{l=\alpha+1}^{n} B_{i l}^{(\alpha)} z_{-l} z_{l}+\gamma_{i}^{(\alpha)} z_{0} \quad(1 \leq i \leq \alpha), \\
z_{k} z_{-k}=\sum_{j=1}^{\alpha} B_{k j}^{(\alpha)} z_{j} z_{-j}+\sum_{l=\alpha+1}^{n} B_{k l}^{(\alpha)} z_{-l} z_{l}+\gamma_{k}^{(\alpha)} z_{0} & (\alpha+1 \leq k \leq n) .
\end{array}
$$

\footnotetext{
${ }^{1}$ In the case $\alpha=0$ we have relations (3.6) and for $\alpha=n$ we obtain the system inverse to (3.6).

${ }^{2}$ In Section 5 the parameter $\alpha$ will characterize a representation type of the discrete series.
} 
Here

$$
\begin{aligned}
& B_{i j}^{(\alpha)}=\frac{b_{i}^{(\alpha)+} b_{j}^{(\alpha)-}}{\left[\varphi_{i j}+1\right]}, \quad B_{i l}^{(\alpha)}=\frac{b_{i}^{(\alpha)+} b_{l}^{(\alpha)+}}{\left[\varphi_{i l}\right]}, \\
& \gamma_{i}^{(\alpha)}=-\left[\varphi_{i, n+1}-\alpha\right] b_{i}^{(\alpha)+} \quad \text { for } 1 \leq i, j \leq \alpha<l \leq n, \\
& B_{k j}^{(\alpha)}=-\frac{b_{k}^{(\alpha)-} b_{j}^{(\alpha)-}}{\left[\varphi_{k j}\right]}, \quad B_{k l}^{(\alpha)}=-\frac{b_{k}^{(\alpha)-} b_{l}^{(\alpha)+}}{\left[\varphi_{k l}-1\right]}, \\
& \gamma_{k}^{(\alpha)}=\left[\varphi_{k, n+1}-\alpha-1\right] b_{k}^{(\alpha)-} \quad \text { for } 1 \leq j \leq \alpha<k, l \leq n,
\end{aligned}
$$

where

$$
\begin{array}{ll}
b_{i}^{(\alpha) \pm}=\left(\prod_{s=1}^{i-1} \frac{\left[\varphi_{i s} \pm 1\right]}{\left[\varphi_{i s}\right]}\right)\left(\prod_{s=\alpha+1}^{n} \frac{\left[\varphi_{i s}\right]}{\left[\varphi_{i s} \pm 1\right]}\right) \quad(1 \leq i \leq \alpha), \\
b_{l}^{(\alpha) \pm}=\left(\prod_{s=1}^{\alpha} \frac{\left[\varphi_{l s}\right]}{\left[\varphi_{l s} \pm 1\right]}\right)\left(\prod_{s=l+1}^{n} \frac{\left[\varphi_{l s} \pm 1\right]}{\left[\varphi_{l s}\right]}\right) \quad(\alpha+1 \leq l \leq n) .
\end{array}
$$

Scheme of proof. The relations (3.9) and (3.10) with the coefficients (3.11)-(3.14) can be proved by induction on $\alpha$. For $\alpha=0$ they coincide with the relations (3.6) $-(3.8)^{3}$. Next we assume that relations (3.9)-(3.14) are valid for $\alpha \geq 1$ and we extract from (3.10) the relation with $k=\alpha+1$ and express in it the term $z_{-\alpha-1} z_{\alpha+1}$ in terms of the elements $z_{1} z_{-1}, \ldots, z_{\alpha+1} z_{-\alpha-1}, z_{-\alpha-2} z_{\alpha+2}, \ldots, z_{-n} z_{n}$; then this expression is substituted in the right side of the rest relations (3.9) and (3.10) and after some algebraic manipulations we obtain the relations (3.9)-(3.14) where $\alpha$ should be replaced by $\alpha+1$.

Using (3.3)-(3.5) and (3.9)-(3.14) we can prove some power relations.

Proposition 2. The following power relations are valid

$$
\begin{aligned}
& z_{i}^{r} z_{-j}^{s}=z_{-j}^{s} z_{i}^{r} \quad \text { for } 1 \leq i, j \leq n, \quad i \neq j \quad \text { and } \quad r, s \in \mathbb{N}, \\
& z_{i}^{r} z_{j}^{s}=z_{j}^{s} z_{i}^{r} \frac{\left[\varphi_{i j}+r\right] !\left[\varphi_{i j}-s\right] !}{\left[\varphi_{i j}\right] !\left[\varphi_{i j}+r-s\right] !} \quad \text { for } \quad 1 \leq i<j \leq n \text { and } \quad r, s \in \mathbb{N}, \\
& z_{-i}^{r} z_{-j}^{s}=z_{-j}^{s} z_{-i}^{r} \frac{\left[\varphi_{i j}\right] !\left[\varphi_{i j}-r+s\right] !}{\left[\varphi_{i j}-r\right] !\left[\varphi_{i j}+s\right] !} \quad \text { for } 1 \leq i<j \leq n \quad \text { and } \quad r, s \in \mathbb{N}, \\
& z_{-i} z_{i}^{r}=z_{i}^{r-1}\left(\sum_{j=1}^{\alpha} B_{i j}^{(\alpha)}(r) z_{j} z_{-j}+\sum_{l=\alpha+1}^{n} B_{i l}^{(\alpha)}(r) z_{-l} z_{l}+\gamma_{i}^{(\alpha)}(r) z_{0}\right) \quad(1 \leq i \leq \alpha), \\
& z_{k} z_{-k}^{r}=z_{-k}^{r-1}\left(\sum_{j=1}^{\alpha} B_{k j}^{(\alpha)}(r) z_{j} z_{-j}+\sum_{l=\alpha+1}^{n} B_{k l}^{(\alpha)}(r) z_{-l} z_{l}+\gamma_{k}^{(\alpha)}(r) z_{0}\right) \\
& (\alpha+1 \leq k \leq n) .
\end{aligned}
$$

Here

$$
\begin{aligned}
& B_{i j}^{(\alpha)}(r)=\frac{[r]}{\left[\varphi_{i j}+r\right]} b_{i}^{(\alpha)+}(r) b_{j}^{(\alpha)-}, \quad B_{i l}^{(\alpha)}(r)=\frac{[r]}{\left[\varphi_{i l}+r-1\right]} b_{i}^{(\alpha)+}(r) b_{l}^{(\alpha)+}, \\
& \gamma_{i}^{(\alpha)}(r)=-[r]\left[\varphi_{i, n+1}-\alpha+r-1\right] b_{i}^{(\alpha)+}(r) \quad(1 \leq i, j \leq \alpha<l \leq n ; \quad r \in \mathbb{N}),
\end{aligned}
$$

\footnotetext{
${ }^{3}$ In this case, the relations (3.9) are absent and, moreover, the first sum in the right side of the relations (3.10) is equal to 0 for $\alpha=0$.
} 


$$
\begin{aligned}
& B_{k j}^{(\alpha)}(r)=-\frac{[r]}{\left[\varphi_{k j}-r+1\right]} b_{k}^{(\alpha)-}(r) b_{j}^{(\alpha)-}, \quad B_{k l}^{(\alpha)}=-\frac{[r]}{\left[\varphi_{k l}-r\right]} b_{k}^{(\alpha)-}(r) b_{l}^{(\alpha)+}, \\
& \gamma_{k}^{(\alpha)}(r)=[r]\left[\varphi_{k, n+1}-\alpha-r\right] b_{k}^{(\alpha)-}(r) \quad(1 \leq j \leq \alpha<k, l \leq n ; \quad r \in \mathbb{N}),
\end{aligned}
$$

where

$$
\begin{aligned}
& b_{i}^{(\alpha)+}(r)=\left(\prod_{s=1}^{i-1} \frac{\left[\varphi_{i s}+r\right]}{\left[\varphi_{i s}+r-1\right]}\right)\left(\prod_{s=\alpha+1}^{n} \frac{\left[\varphi_{i s}+r-1\right]}{\left[\varphi_{i s}+r\right]}\right) \quad(1 \leq i \leq \alpha), \\
& b_{j}^{(\alpha)-}=\left(\prod_{s=1}^{j-1} \frac{\left[\varphi_{j s}-1\right]}{\left[\varphi_{j s}\right]}\right)\left(\prod_{s=\alpha+1}^{n} \frac{\left[\varphi_{j s}\right]}{\left[\varphi_{j s}-1\right]}\right) \quad(1 \leq j \leq \alpha), \\
& b_{k}^{(\alpha)-}(r)=\left(\prod_{s=1}^{\alpha} \frac{\left[\varphi_{k s}-r+1\right]}{\left[\varphi_{k s}-r\right]}\right)\left(\prod_{s=k+1}^{n} \frac{\left[\varphi_{k s}-r\right]}{\left[\varphi_{k s}-r+1\right]}\right) \quad(\alpha+1 \leq k \leq n), \\
& b_{l}^{(\alpha)+}=\left(\prod_{s=1}^{\alpha} \frac{\left[\varphi_{l s}\right]}{\left[\varphi_{l s}+1\right]}\right)\left(\prod_{s=l+1}^{n} \frac{\left[\varphi_{l s}+1\right]}{\left[\varphi_{l s}\right]}\right) \quad(\alpha+1 \leq l \leq n) .
\end{aligned}
$$

Here in (3.16), (3.17) and thoughtout in Section 4 we use the short notation of the $q$-factorial $[x+n] !=[x+n][x+n-1] \cdots[x+1][x]$ ! instead the $q$-Gamma function, $[x+n] ! \equiv \Gamma_{q}([x+n+1])$.

Sketch of proof. The relations (3.15)-(3.17) are a direct consequence of the relations (3.3)(3.5). Relations (3.18) and (3.19) with the coefficients (3.20)-(3.25) are proved by induction on $r$ using the initial relations (3.9) and (3.10) with the coefficients (3.11)-(3.14) for $r=1$.

\section{Shapovalov forms on $Z_{q}(\mathfrak{g l}(n+1), \mathfrak{g l}(n))$}

Let us consider properties of the $Z$-algebra $Z_{q}(\mathfrak{g l}(n+1), \mathfrak{g l}(n))$ with respect to the involutions $\star$ $(2.26)-(2.28)$, and $*(2.30)-(2.33)$.

Proposition 3. The $Z$-algebra $Z_{q}(\mathfrak{g l}(n+1), \mathfrak{g l}(n))$ is invariant with respect to the involutions $\star$ and $*$, besides

$$
z_{0}^{\star}=z_{0}, \quad z_{i}^{\star}=z_{-i} \quad \text { for } \quad i= \pm 1, \pm 2, \ldots, \pm n,
$$

and

$$
z_{0}^{*}=z_{0}, \quad z_{i}^{*}=-z_{-i} \quad \text { for } i= \pm 1, \pm 2, \ldots, \pm n .
$$

Proof. Because the extremal projector $p=p(\mathfrak{g l}(n))$ is $*$-invariant, $p^{*}=p$ for $*=\star, *$ (see the formula (2.29)), it turns out that

$$
z_{i}^{*}=p e_{i, n+1}^{*} p, \quad z_{-i}^{*}=p e_{n+1, i}^{*} p \quad \text { for } i=1,2, \ldots, n .
$$

If $q$ is circular, then $e_{i, n+1}^{*}= \pm e_{n+1, i}, e_{n+1, i}^{*}= \pm e_{i, n+1}$, where the plus belongs to the compact case and the minus belongs to the noncompact case, and we obtain the formulas (4.1) and (4.2).

If $q$ is real, then $e_{n+1, i}^{*}= \pm e_{i, n+1}^{\prime}, e_{i, n+1}^{*}= \pm e_{n+1, i}^{\prime}$ where the root vectors $e_{i, n+1}^{\prime}$ and $e_{n+1, i}^{\prime}$ are obtained from (2.10) by the replacement $q^{ \pm 1} \rightarrow q^{\mp 1}$. Let us consider the difference $z_{-i}^{*}-z_{i}=$ $p\left(e_{i, n+1}^{\prime}-e_{i, n+1}\right) p$ for $\left.1 \leq i \leq n\right)$. Substituting here (see the formulas $(2.11)$ )

$$
e_{i, n+1}^{\prime}=e_{i n}^{\prime} e_{n, n+1}-q^{-1} e_{n, n+1} e_{i n}^{\prime}, \quad e_{i, n+1}=e_{i n} e_{n, n+1}-q e_{n, n+1} e_{i n},
$$


and using the annihilation properties of the projector $p$ (see (2.25)) we obtain $z_{-i}^{*}-z_{i}=p\left(e_{i n}^{\prime}-\right.$ $\left.e_{i n}\right) e_{n, n+1} p$. In a similar way, using explicit formulas of type (4.3) for the generators $e_{i n}^{\prime}$ and $e_{i n}$, we obtain $z_{-i}^{*}-z_{i}=p\left(e_{i, n-1}^{\prime}-e_{i, n-1}\right) e_{n-1, n} e_{n, n+1} p$. By proceeding as above, we have $z_{-i}^{*}-$ $z_{i}=p\left(e_{i, i+1}-e_{i, i+1}\right) e_{i+1, i+2} e_{i+2, i+3} \cdots e_{n-1, n} e_{n, n+1} p=0$. In a similar way, we prove that $z_{i}^{*}-z_{-i}=0$.

The $Z$-algebra $Z_{q}(\mathfrak{g l}(n+1), \mathfrak{g l}(n))$ with the involution $\star$ is called the compact real form and is denoted by the symbol $Z_{q}^{(c)}(\mathfrak{g l}(n+1), \mathfrak{g l}(n))$. The noncompact real form on $Z_{q}(\mathfrak{g l}(n+1), \mathfrak{g l}(n))$ is defined by the involution $*$ and is denoted by the symbol $Z_{q}^{(n c)}(\mathfrak{g l}(n+1), \mathfrak{g l}(n))$.

Let $p^{(\alpha)}$ be an extremal projector for $Z_{q}(\mathfrak{g l}(n+1), \mathfrak{g l}(n))$ satisfying the relations

$$
\begin{aligned}
& z_{-i} p^{(\alpha)}=p^{(\alpha)} z_{i} \quad \text { for } i=1,2, \ldots, \alpha \\
& z_{k} p^{(\alpha)}=p^{(\alpha)} z_{-k} \quad \text { for } \quad k=\alpha+1, \alpha+2, \ldots, n \\
& {\left[e_{i i}, p^{(\alpha)}\right]=0 \quad \text { for } \quad i=1,2, \ldots, n}
\end{aligned}
$$

The extremal projector $p^{(\alpha)}$ depends on the index $\alpha$ that defines what elements are considered as "raising" and what elements are considered as "lowering", i.e. in our case the elements $z_{-1}, z_{-2}, \ldots, z_{-\alpha}, z_{\alpha+1}, \ldots, z_{n}$ are raising and the elements $z_{1}, z_{2}, \ldots, z_{\alpha}, z_{-\alpha-1}, \ldots, z_{-n}$ are lowering. It should be stressed that the "raising" and "lowering" subsets generate disjoint subalgebras in $Z_{q}(\mathfrak{g l}(n+1), \mathfrak{g l}(n))$. The operator $p^{(\alpha)}$ can be constructed in an explicit form.

Let us introduce on $Z_{q}^{(n c)}(\mathfrak{g l}(n+1), \mathfrak{g l}(n))$ the following sesquilinear Shapovalov form [16]. For any elements $x, y \in Z_{q}^{(n c)}(\mathfrak{g l}(n+1), \mathfrak{g l}(n))$ we set

$$
B^{(\alpha)}(x, y)=p^{(\alpha)} y^{*} x p^{(\alpha)} \text {. }
$$

Therefore, the Shapovalov form also depends on the index $\alpha(\alpha=0,1,2, \ldots, n)$. We fix $\alpha$ $(\alpha=0,1,2, \ldots, n)$ and for each set of nonnegative integers $\{r\}=\left(r_{1}, r_{2}, \ldots, r_{n}\right)$ introduce a vector $v_{\{r\}}^{(\alpha)}$ in the space $Z_{q}^{(n c)}(\mathfrak{g l}(n+1), \mathfrak{g l}(n))$ by the formula

$$
v_{\{r\}}^{(\alpha)}=z_{\alpha}^{r_{\alpha}} \cdots z_{1}^{r_{1}} z_{-\alpha-1}^{r_{\alpha}+1} \cdots z_{-n}^{r_{n}} .
$$

Theorem 2. For each fixed $\alpha(\alpha=0,1,2, \ldots, n)$ the vectors $\left\{v_{\{r\}}^{(\alpha)}\right\}$ are pairwise orthogonal with respect to the Shapovalov form (4.4)

$$
B^{(\alpha)}\left(v_{\{r\}}^{(\alpha)}, v_{\left\{r^{\prime}\right\}}^{(\alpha)}\right)=\delta_{\{r\},\left\{r^{\prime}\right\}} B^{(\alpha)}\left(v_{\{r\}}^{(\alpha)}, v_{\{r\}}^{(\alpha)}\right) .
$$

and

$$
\begin{aligned}
B^{(\alpha)}\left(v_{\{r\}}^{(\alpha)}, v_{\{r\}}^{(\alpha)}\right)= & \left(\prod_{i=1}^{\alpha} \frac{\left[r_{i}\right] !\left[\varphi_{i, n+1}-\alpha+r_{i}-1\right] !}{\left[\varphi_{i, n+1}-\alpha-1\right] !} \prod_{l=\alpha+1}^{n} \frac{\left[r_{l}\right] !\left[\varphi_{n+1, l}+\alpha+r_{l}\right] !}{\left[\varphi_{n+1, l}+\alpha\right] !}\right. \\
& \times \prod_{1 \leq i<j \leq \alpha} \frac{\left[\varphi_{i j}+r_{i}-r_{j}\right] !\left[\varphi_{i j}-1\right] !}{\left[\varphi_{i j}+r_{i}\right] !\left[\varphi_{i j}-r_{j}-1\right] !} \prod_{\alpha+1 \leq k<l \leq n} \frac{\left[\varphi_{k l}-r_{k}+r_{l}\right] !\left[\varphi_{k l}-1\right] !}{\left[\varphi_{k l}-r_{k}-1\right] !\left[\varphi_{k l}+r_{l}\right] !} \\
& \left.\times \prod_{1 \leq i \leq \alpha<l \leq n} \frac{\left[\varphi_{i l}+r_{i}-1\right] !\left[\varphi_{i l}+r_{l}-1\right] !\left[\varphi_{i l}\right]}{\left[\varphi_{i l}+r_{i}+r_{l}\right] !\left[\varphi_{i l}-1\right] !}\right) z_{0}^{(\alpha)}
\end{aligned}
$$

where $z_{0}^{(\alpha)} \equiv p^{(\alpha)}$. 
As a consequence of this theorem we obtain that the Shapovalov form is not degenerate on a subspace of $Z_{q}^{(n c)}(\mathfrak{g l}(n+1), \mathfrak{g l}(n))$, generated by the vectors of form (4.5).

In the case of the compact $Z$-algebra $Z_{q}^{(c)}(\mathfrak{g l}(n+1), \mathfrak{g l}(n))$ the Shapovalov form $B(x, y)$ is defined by formula (4.4) where $\alpha=0, p^{(0)}$ is the standard extremal projector of the quantum algebra $U_{q}(\mathfrak{g l}(n+1))$ and the involution is given by formulas (4.1). It is not difficult to see that

$$
B\left(v_{\{r\}}, v_{\left\{r^{\prime}\right\}}\right)=\delta_{\{r\},\left\{r^{\prime}\right\}} B\left(v_{\{r\}}, v_{\{r\}}\right) .
$$

where $v_{\{r\}}:=v_{\{r\}}^{(0)}$ and

$$
\begin{aligned}
B\left(v_{\{r\}}, v_{\{r\}}\right) & =(-1)^{\sum_{i=1}^{n} r_{i}} B^{(0)}\left(v_{\{r\}}^{(0)}, v_{\{r\}}^{(0)}\right) \\
& =\left(\prod_{l=1}^{n} \frac{\left[r_{l}\right] !\left[\varphi_{l, n+1}-1\right] !}{\left[\varphi_{l, n+1}-r_{l}-1\right] !} \prod_{1 \leq k<l \leq n} \frac{\left[\varphi_{k l}-r_{k}+r_{l}\right] !\left[\varphi_{k l}-1\right] !}{\left[\varphi_{k l}-r_{k}-1\right] !\left[\varphi_{k l}+r_{l}\right] !}\right) z_{0}^{(0)}
\end{aligned}
$$

\section{Discrete series of representations for $U_{q}(u(n, 1))$}

As in the classical case [9] each Hermitian irreducible representation of the discrete series for the noncompact quantum algebra $U_{q}(u(n, 1))$ is defined uniquely by some extremal vector $|x w\rangle$, the vector of extremal weight ${ }^{4}$. This vector should be the highest vector with respect to the compact subalgebra $U_{q}(u(n)) \oplus U_{q}(u(1))$. Since the quantum algebra $U_{q}(u(1))$ is generated only by one Cartan element $q^{e_{n+1, n+1}}$, the vector $|x w\rangle$ should be annihilated by the raising generators $e_{i j}$ $(1 \leq i<j \leq n)$ of the compact subalgebra $U_{q}(u(n))$. So the vector $|x w\rangle$ satisfies the relations

$$
\begin{aligned}
& e_{i i}|x w\rangle=\mu_{i}|x w\rangle \quad(i=1,2, \ldots, n+1), \\
& e_{i j}|x w\rangle=0 \quad(1 \leq i<j \leq n),
\end{aligned}
$$

where the weight components $\mu_{i}(i=1,2, \ldots, n)$ are integers subjected to the condition $\mu_{1} \geq$ $\mu_{2} \geq \cdots \geq \mu_{n}$. Such weights can be compared with respect to standard lexicographic ordering, namely, $\mu>\mu^{\prime}$, where $\mu=\left(\mu_{1}, \mu_{2}, \ldots, \mu_{n}\right)$ and $\mu^{\prime}=\left(\mu_{1}^{\prime}, \mu_{2}^{\prime}, \ldots, \mu_{n}^{\prime}\right)$, if a first nonvanishing component of the difference $\mu-\mu^{\prime}$ is positive.

The component $\mu_{n+1}$ is also an integer. In the general case of finite-dimensional irreducible representations of the compact quantum algebra $U_{q}(u(n)) \oplus U_{q}(u(1))$, the weights $\mu=\left(\mu_{1}, \mu_{2}\right.$, $\left.\ldots, \mu_{n}\right)$ and $\mu_{n+1}$ are not ordering. If we choose some ordering for these weights, for example, $\left(\mu_{1}, \ldots, \mu_{\alpha}, \mu_{n+1}, \mu_{\alpha+1}, \ldots, \mu_{n}\right)$, then such $n+1$-component weights can be compared.

The extremal vector $|x w\rangle$ has the minimal weight $\Lambda_{n+1}^{(\alpha)}:=\left(\lambda_{1, n+1}, \lambda_{2, n+1}, \ldots, \lambda_{n+1, n+1}\right)$ where $\lambda_{i, n+1}:=\mu_{i}(i=1,2, \ldots, \alpha), \lambda_{\alpha+1, n+1}:=\mu_{n+1}, \lambda_{l+1, n+1}:=\mu_{l}(l=\alpha+1, \ldots, n)$. The vector $\left|\Lambda_{n+1}^{(\alpha)}\right\rangle:=|x w\rangle$ with the weight $\Lambda_{n+1}^{(\alpha)}$ satisfies the relations

$$
\begin{aligned}
& z_{-i}\left|\Lambda_{n+1}^{(\alpha)}\right\rangle=0, \quad \text { for } i=1,2, \ldots, \alpha, \\
& z_{k}\left|\Lambda_{n+1}^{(\alpha)}\right\rangle=0, \quad \text { for } \quad k=\alpha+1, \alpha+2, \ldots, n .
\end{aligned}
$$

It is evident that any highest weight vector $\left.\mid \Lambda_{n+1}^{(\alpha)} ; \Lambda_{n}\right)$ with respect to the compact subalgebra $U_{q}(u(n))$ has the form

$$
\left.\mid \Lambda_{n+1}^{(\alpha)} ; \Lambda_{n}\right)=z_{\alpha}^{r_{\alpha}} \cdots z_{1}^{r_{1}} z_{-\alpha-1}^{r_{\alpha+1}} \cdots z_{-n}^{r_{n}}\left|\Lambda_{n+1}^{(\alpha)}\right\rangle .
$$

\footnotetext{
${ }^{4}$ We assume that the vector $|x w\rangle$ is orthonormal, $\langle x w \mid x w\rangle=1$.
} 
Here the integers $\{r\}$ are defined by the weights $\Lambda_{n+1}^{(\alpha)}=\left(\lambda_{1, n+1}, \lambda_{2, n+1}, \ldots, \lambda_{n+1, n+1}\right)$, where $\lambda_{i, n+1} \geq \lambda_{i+1, n+1}(i=1,2, \ldots, n)$, and $\Lambda_{n}=\left(\lambda_{1 n}, \lambda_{2 n}, \ldots, \lambda_{n n}\right)$, where $\lambda_{i n} \geq \lambda_{i+1, n}(i=$ $1,2, \ldots, n-1)$, namely,

$$
\begin{aligned}
& r_{i}=\lambda_{i n}-\lambda_{i, n+1} \quad(i=1, \ldots, \alpha), \\
& r_{l}=\lambda_{l+1, n+1}-\lambda_{l n} \quad(l=\alpha+1, \ldots, n) .
\end{aligned}
$$

If we would like to calculate the scalar product of the two vectors (5.1) then using the results for the Shapovalov form (4.6), (4.7) we obtain

$$
\begin{aligned}
& \left(\Lambda_{n} ; \Lambda_{n+1}^{(\alpha)} \mid \Lambda_{n+1}^{(\alpha)} ; \Lambda_{n}^{\prime}\right)=\delta_{\Lambda_{n}, \Lambda_{n}^{\prime}}\left(\Lambda_{n} ; \Lambda_{n+1}^{(\alpha)} \mid \Lambda_{n+1}^{(\alpha)} ; \Lambda_{n}\right) \\
& \left(\Lambda_{n} ; \Lambda_{n+1}^{(\alpha)} \mid \Lambda_{n+1}^{(\alpha)} ; \Lambda_{n}\right)=\left.B^{(\alpha)}\left(v_{\{r\}}^{(\alpha)}, v_{\{r\}}^{(\alpha)}\right)\right|_{\Lambda_{n+1}^{(\alpha)}},
\end{aligned}
$$

where the symbol $\left.\right|_{\Lambda_{n+1}^{(\alpha)}}$ means that we specialize the Shapovalov form (4.7) for the extremal weight $\Lambda_{n+1}$, that is we replace the Cartan elements $e_{i i}, e_{j j}$ in the functions $\varphi_{i j}$ by the corresponding components $\lambda_{i, n+1}, \lambda_{j, n+1}$ and $z_{0}$ by 1 .

From the condition that

$$
\left(\Lambda_{n} ; \Lambda_{n+1}^{(\alpha)} \mid \Lambda_{n+1}^{(\alpha)} ; \Lambda_{n}\right)>0
$$

we find all admissible highest weights $\Lambda_{n}$ of the compact subalgebra $U_{q}(u(n))$. The result is formulated as the theorem

Theorem 3. 1) Every Hermitian irreducible representation of the discrete series for the noncompact quantum algebra $U_{q}(u(n, 1))$ with the extremal weight $\Lambda_{n+1}^{(\alpha)}=\left(\lambda_{1, n+1}, \ldots, \lambda_{n+1, n+1}\right)$, where the integers $\lambda_{i, n+1}$ satisfy the inequalities $\lambda_{i, n+1} \geq \lambda_{i+1, n+1}(i=1,2, \ldots, n)$, under the restriction $U_{q}(u(n, 1)) \downarrow U_{q}(u(n))$ contains all multiplicity free irreducible representations of the compact subalgebra $U_{q}(u(n))$ with the highest weights $\Lambda_{n}=\left(\lambda_{1 n}, \lambda_{2 n}, \ldots, \lambda_{n n}\right)$ satisfying the conditions:

$$
\begin{aligned}
& \lambda_{1 n} \geq \lambda_{1, n+1} \geq \lambda_{2, n} \geq \lambda_{2, n+1} \geq \cdots \geq \lambda_{\alpha n} \geq \lambda_{\alpha, n+1}, \\
& \lambda_{\alpha+2, n+1} \geq \lambda_{\alpha+1, n} \geq \lambda_{\alpha+3, n+1} \geq \cdots \geq \lambda_{n+1, n+1} \geq \lambda_{n n} .
\end{aligned}
$$

2) The vectors

$$
\left|\Lambda_{n+1}^{(\alpha)} ; \Lambda_{n}\right\rangle=F_{-}^{(\alpha)}\left(\Lambda_{n} ; \Lambda_{n+1}^{(\alpha)}\right)\left|\Lambda_{n+1}^{(\alpha)}\right\rangle,
$$

where the "lowering" operator $F_{-}^{(\alpha)}\left(\Lambda_{n} ; \Lambda_{n+1}^{(\alpha)}\right)$ is given by

$$
\begin{aligned}
F_{-}^{(\alpha)}\left(\Lambda_{n} ; \Lambda_{n+1}^{(\alpha)}\right)= & N^{(\alpha)}\left(\Lambda_{n} ; \Lambda_{n+1}^{(\alpha)}\right) z_{\alpha}^{\lambda_{\alpha n}-\lambda_{\alpha, n+1}} \cdots z_{1}^{\lambda_{1 n}-\lambda_{1, n+1}} \\
& \times z_{-\alpha-1}^{\lambda_{\alpha+2, n+1}-\lambda_{\alpha+1, n}} \cdots z_{-n}^{\lambda_{n+1, n+1}-\lambda_{n n}}
\end{aligned}
$$

for all highest wights $\Lambda_{n}=\left(\lambda_{1 n}, \lambda_{2 n}, \ldots, \lambda_{n n}\right)$ constrained by the conditions (5.2) form an orthonormal basis in the space of the highest vectors with respect to the compact subalgebra $U_{q}(u(n))$. Here in (5.3) the normalized factor $N^{(\alpha)}\left(\Lambda_{n} ; \Lambda_{n+1}^{(\alpha)}\right)$ is given as follows:

$$
\begin{aligned}
N^{(\alpha)}\left(\Lambda_{n} ; \Lambda_{n+1}^{(\alpha)}\right) & =\left(\Lambda_{n} ; \Lambda_{n+1}^{(\alpha)} \mid \Lambda_{n+1}^{(\alpha)} ; \Lambda_{n}\right)^{-\frac{1}{2}} \\
& =\left\{\prod_{i=1}^{\alpha} \frac{\left[l_{i, n+1}-l_{\alpha+1, n+1}-2 \alpha+n-1\right] !}{\left[l_{i n}-l_{i, n+1}\right] !\left[l_{i, n}-l_{\alpha+1, n+1}-2 \alpha+n-1\right] !}\right.
\end{aligned}
$$




$$
\begin{aligned}
& \times \prod_{l=\alpha+1}^{n} \frac{\left[l_{\alpha+1, n+1}-l_{l+1, n+1}+2 \alpha-n-1\right] !}{\left[l_{l+1, n+1}-l_{l n}-1\right] !\left[l_{\alpha+1, n+1}-l_{l n}+2 \alpha-n\right] !} \\
& \times \prod_{1 \leq i<j \leq \alpha} \frac{\left[l_{i n}-l_{j, n+1}\right] !\left[l_{i, n+1}-l_{j n}-1\right] !}{\left[l_{i n}-l_{j n}\right] !\left[l_{i, n+1}-l_{j, n+1}-1\right] !} \\
& \times \prod_{\alpha+1 \leq k<l \leq n} \frac{\left[l_{k n}-l_{l+1, n+1}-2\right] !\left[l_{k+1, n+1}-l_{l n}+1\right] !}{\left[l_{k n}-l_{l n}\right] !\left[l_{k+1, n+1}-l_{l+1, n+1}-1\right] !} \\
& \left.\times \prod_{1 \leq i \leq \alpha<l \leq n} \frac{\left[l_{i n}-l_{l n}\right] !\left[l_{i, n+1}-l_{l+1, n+1}-2\right] !}{\left[l_{i n}-l_{l+1, n+1}-2\right] !\left[l_{i, n+1}-l_{l n}-1\right] !\left[l_{i, n+1}-l_{l+1, n+1}-1\right]}\right\}^{\frac{1}{2}}
\end{aligned}
$$

$\left(l_{s r}:=\lambda_{s r}-s\right.$ for $\left.s=1,2, \ldots, r ; r=n, n+1\right)$.

The first part of the theorem coincides with the classical Gelfand-Graev case [5, 6] for the noncompact Lie algebra $u(n, 1)$. Using analogous construction of the Gelfand-Tsetlin basis for the compact quantum algebra $U_{q}(u(n))$ [14] we obtain a $q$-analog of the Gelfand-Graev-Tsetlin basis for $U_{q}(u(n, 1))$. Namely, in the $U_{q}(u(n, 1))$-module with the extremal weight $\Lambda_{n+1}^{(\alpha)}$ there is an orthogonal Gelfand-Graev-Tsetlin basis consisting of all vectors of the form

$$
\left.|\Lambda\rangle:=\mid \begin{array}{c}
\Lambda_{n+1}^{(\alpha)} \\
\Lambda_{n} \\
\cdots \\
\Lambda_{2} \\
\Lambda_{1}
\end{array}\right\}=F_{-}\left(\Lambda_{1} ; \Lambda_{2}\right) F_{-}\left(\Lambda_{2} ; \Lambda_{3}\right) \cdots F_{-}\left(\Lambda_{n-1} ; \Lambda_{n}\right)\left|\Lambda_{n+1}^{(\alpha)} ; \Lambda_{n}\right\rangle
$$

where $\Lambda_{j}=\left(\lambda_{1 j}, \lambda_{2 j}, \ldots, \lambda_{j j}\right)(j=1,2, \ldots, n)$ and the numbers $\lambda_{i j}$ satisfy the standard "between conditions" for the quantum algebra $U_{q}(u(n))$, i.e.

$$
\lambda_{i, j+1} \geq \lambda_{i j} \geq \lambda_{i+1, j+1} \quad \text { for } 1 \leq i \leq j \leq n-1 .
$$

The lowering operators $F_{-}\left(\Lambda_{k} ; \Lambda_{k+1}\right)(k=1,2, \ldots, n-1)$ are given by (see $\left.[14,15]\right)$

$$
\begin{aligned}
F_{-}\left(\Lambda_{k} ; \Lambda_{k+1}\right)= & N\left(\Lambda_{k} ; \Lambda_{k+1}\right) p\left(U_{q}(u(k))\right) \prod_{i=1}^{k}\left(e_{k+1 i}\right)^{\lambda_{i k+1}-\lambda_{i k}}, \\
N\left(\Lambda_{k} ; \Lambda_{k+1}\right)= & \left\{\prod_{i=1}^{k} \frac{\left[l_{i k}-l_{k+1, k+1}-1\right] !}{\left[l_{i, k+1}-l_{i k}\right] !\left[l_{i, k+1}-l_{k+1, k+1}-1\right] !}\right. \\
& \left.\times \prod_{1 \leq i<j \leq k} \frac{\left[l_{i, k+1}-l_{j k}\right] !\left[l_{i k}-l_{j, k+1}-1\right] !}{\left[l_{i k}-l_{j k}\right] !\left[l_{i, k+1}-l_{j, k+1}-1\right] !}\right\}^{\frac{1}{2}},
\end{aligned}
$$

where $l_{i j}:=\lambda_{i j}-i$ for $1 \leq i \leq j \leq n-1$. This explicit construction allows one to obtain formulas for actions of the $U_{q}(u(n, 1))$-generators. These results will be presented elsewhere.

\section{Summary}

Thus, we obtain the explicit description of the Hermitian irreducible representations of the discrete series for the noncompact quantum algebra $U_{q}(u(n, 1))$ by the reduction $Z$-algebras for description of which we used the standard extremal projectors.

Next step: to obtain analogous results for $U_{q}(u(n, 2))$. For this aim we need to construct the extremal projector $p^{(\alpha)}$ which is expressed in terms of the $Z$-algebra $Z_{q}(\mathfrak{g l}(n+1), \mathfrak{g l}(n))$.

Final aim: to consider the general case $U_{q}(u(n, m))$. In this case, extremal projectors of new type will be used. 


\section{Acknowledgments}

The paper has been supported by grant RFBR-08-01-00392 (R.M.A., V.N.T.) and by grant RFBR-09-01-93106-NCNIL-a (V.N.T.). The fifth author would like to thank Department of Mathematics, Faculty of Nuclear Sciences and Physical Engineering, Czech Technical University in Prague for hospitality.

\section{References}

[1] Gel'fand I.M., Tsetlin M.L., Finite-dimensional representations of unimodular matrices group, Doklady Akad. Nauk SSSR 71 (1950), 825-828 (in Russian).

[2] Nagel J.G., Moshinsky M., Operators that lower or raise the irreducible vector spaces of $U_{n-1}$ contained in an irreducible vector space of $U_{n}, J$. Math. Phys. 6 (1965), 682-694.

[3] Hou P.-Y., Orthonormal bases and infinitesimal operators of the irreducible representations of group $U_{n}$, Sci. Sinica 15 (1966), 763-772.

[4] Asherova R.M., Smirnov Yu.F., Tolstoy V.N., Projection operators for the simple Lie groups. II. General scheme for construction of lowering operators. The case of the group SU(n), Teoret. Mat. Fiz. 15 (1973), no. 1, 107-119 (in Russian).

[5] Gelfand I.M., Graev M.I., Finite-dimensional irreducible representations of unitary and general linear groups and related special functions, Izv. Akad. Nauk SSSR Ser. Mat. 29 (1965), no. 6, 1329-1356 (English transl.: Amer. Math. Soc. Transl. (2) 64 (1967), 116-146).

[6] Barut A., Rączka R., Theory of group representations and applications, Polish Scientific Publishers, Warsaw, 1977.

[7] Enright T.J., Varadarajan V.S., On an infinitesimal characterization of the discrete series, Ann. of Math. (2) 102 (1975), 1-15.

[8] Molev A.I., Unitarizability of some Enright-Varadarajan $u(p, q)$-modules, in Topics in Representation Theory, Adv. Soviet Math., Vol. 2, Amer. Math. Soc., Providence, RI, 1991, 199-219.

[9] Mickelsson J., A description of discrete series using step algebra, Math. Scand. 41 (1977), 63-78.

[10] Smirnov Yu.F., Kharitonov Yu.I., Noncompact quantum algebra $u_{q}(2,1)$ : positive discrete series of irreducible representations, in Proceedings of International Symposium "Symmetries in Science XI" (July 2003, Bregenz, Austria), Editors B. Gruber, G. Marmo and N. Ioshinaga, Kluwer Acad. Publ., Dordrecht, 2004, 505-526, math.QA/0311283.

[11] Smirnov Yu.F., Kharitonov Yu.I., Asherova R.M., Noncompact quantum algebra $u_{q}(2,1)$ : intermediate discrete series of unitary irreducible representations, Phys. Atomic Nuclei 69 (2006), 1045-1057.

[12] Groza V.A., Iorgov N.Z., Klimyk A.U., Representations of the quantum algebra $U_{q}\left(u_{n, 1}\right)$, Algebr. Represent. Theory 3 (2000), 105-130, math.QA/9805032.

[13] Guizzi V., A classification of unitary highest weight modules of the quantum analogue of the symmetric pair $\left(A_{n}, A_{n-1}\right)$, J. Algebra 192 (1997), 102-129.

[14] Tolstoy V.N., Extremal projectors for quantized Kac-Moody superalgebras and some of their applications, in Quantum Groups (Clausthal, 1989), Lecture Notes in Phys., Vol. 370, Springer, Berlin, 1990, 118-125.

[15] Tolstoy V.N., Projection operator method for quantum groups, in Special Functions 2000: Current Perspective and Future Directions (Tempe, AZ), NATO Sci. Ser. II Math. Phys. Chem., Vol. 30, Kluwer Acad. Publ., Dordrecht, 2001, 457-488, math.QA/0104045.

[16] Shapovalov N.N., On a bilinear form on the universal enveloping algebra of a complex semisimple Lie algebra, Funct. Anal. Appl. 6 (1972), 307-312. 